\title{
Ectopic Gene Expression to Restore the Ascorbate Biosynthesis Pathway in Vertebrates Lacking Functional Gulo
}

\author{
Cao CR, Kelly R, Igdoura SA and Schellhorn HE* \\ Department of Biology, McMaster University, Hamilton, Ontario Canada \\ *Corresponding author: Herb Schellhorn, Department of Biology, McMaster University, \\ Hamilton, Ontario Canada, Email: schell@mcmaster.ca
}

\section{Review Article \\ Volume 2 Issue 1}

Received Date: July 30, 2018

Published Date: August 21, 2018

DOI: $10.23880 /$ ggtij-16000108

\begin{abstract}
Background: Vitamin C (VC) deficiency is due to loss of the gene coding for t-gulono- $\gamma$-lactone oxidase (Gulo). VC deficiency is an excellent candidate for therapeutic intervention by ectopic gene expression because a single gene mutation is responsible for the condition and, it is known, a priori, that supplementation effectively rescues the deficiency (at least with respect to the development of scurvy). There are several strategies to ectopically express exogenous genes or silence endogenous genes including viruses and nucleic acids, to ensure that the protein products encoded by exogenous genes are present in the cell, or those encoded by endogenous genes to be silenced are absent.

Objective: We review strategies to restore ascorbate biosynthesis in vertebrates. We will discuss the physiology of vitamin $\mathrm{C}$ and evaluate the potential of the different methods for the restoration of Gulo expression, and subsequently the endogenous production of VC.
\end{abstract}

Methods: Studies that have targeted manipulation of vitamin C synthesis in vertebrates, particularly the mouse model, over the last 20 years were examined.

Results: Vectors of ectopic gene expression were identified as nucleic acids such as linear and plasmid DNA, mRNA, as well as viruses such as adenoviruses and lentiviruses. Direct enzyme replacement was identified as another method of Gulo expression.

Conclusion: Ectopic gene expression can be used to rescue metabolic deficiency in VC synthesis using several different modes of delivery. New technologies that directly modify genomes (e.g. CRISPR) that have not yet been applied to this physiological deficiency may allow new investigations into VC physiology.

Keywords: Ascorbic acid; Vitamin C; Gulonolactone oxidase; Biosynthesis; Knockout; Gene expression; Vectors; Plasmids 


\section{Genomics \& Gene Therapy International Journal}

Abbreviations: ASC - ı-Ascorbic Acid/Ascorbate; B6 C57BL/6 mouse strain; DHASC - Dehydro ascorbate; ECD - Electrochemical detector; EDTA - Ethylene diamine tetraacetic acid; FGAd - First generation adenovirus; Gulo - Gene coding for t-gulono- $\gamma$-lactone oxidase; HDAd Helper-dependent adenovirus; HEPG2 - Human hepatocellular carcinoma cell line; HEK293Cre4 - Human embryonic kidney cell line; GL - ı-gulono- $\gamma$-lactone; mCMV - Murine cytomegalovirus; PEPCK - Phosphoenol pyruvate carboxy kinase; pCMV - Plasmid containing the cytomegalovirus promoter; ROS - Reactive oxygen species; VC - Vitamin C.

\section{Introduction}

Most animals, with exception of certain vertebrate taxa, are able to synthesize ASC endogenously. The animals that cannot synthesize VC, including humans, lack a key enzyme required for VC biosynthesis pathway in the liver, from the starting molecule glucose [1,2]. Without dietary supplementation, symptoms of scurvy develop, which can be a fatal condition if not treated. In addition, ASC is a cofactor for many enzymes responsible for maintaining the integrity of connective tissue in the body, as well as neurological and immune health. A loss of function mutation in the Gulo gene, which codes for the tgulono- $\gamma$-lactone oxidase enzyme that converts GL into ASC, the active form of VC used by the body [3] results in a disrupted ASC biosynthetic pathway. This final step in the ascorbic acid biosynthesis pathway is the rate-limiting step and thus restoring functionality of Gulo could potential prevent scurvy and, more importantly other pathophysiological conditions associated with VC deficiency such as heart disease and cancer.

The common mouse, Mus musculus, is able to synthesize endogenous ASC in the liver and does not require supplements of $\mathrm{VC}$ to stay healthy. The genetically engineered Gulo (-/-) knockout mouse that lacks functional Gulo enzyme requires dietary supplementation to survive, making it a model of choice for studying the effects of VC deficiency in humans. Although VC can be obtained through food, the ability to synthesize endogenous VC may have therapeutic benefits for the treatment of cancer, oxidative stress and cardiovascular disease, [4-6] as its levels in the body does not rely on diet, but remain at constant levels with possible physiological upregulation.

Gulo function can be potentially restored by ectopic gene expression through the use of helper-dependent adenoviruses (HDAd), as indicated by a rise in the serum ascorbate levels of Gulo (-/-) knockout mice after administration of the HDAd-mCMV-Gulo viruses [7]. Due to the cost and complexity of manufacturing viruses, plasmids and other nucleic acids are a promising nonviral alternative for gene therapy [8]. In particular, plasmids are of interest, due to their ease of production and relative stability [9]. Rats express exogenous luciferase in pCMV plasmids carrying a luciferase transgene construct when these plasmids are administered into the abdominal cavity [9]. In this review, we will review the physiology of vitamin C metabolism, as well as the complications of its deficiency and the different methods of ectopic gene expression, in the context of augmenting the expression of functional Gulo in the mouse model.

\section{Vitamin C}

VC is a water-soluble vitamin that is abundant in many fruits and vegetables, especially citrus fruits. Comprised of different chemical species, ASC is the reduced, most common form of vitamin C [10]. It has a short half-life and is easily oxidized, making it somewhat difficult to measure [11]. In several different tissues, ASC is transported intercellularly via an active sodiumdependent vitamin C transporter (SVCT) $[12,13]$. The concentration of ASC in the serum rises in a dosedependent manner with oral intake up to a concentration of approximately $200 \mathrm{umol} / \mathrm{L}$, while the normal physiological range is approximately $60-100 \mathrm{mmol} / \mathrm{L}$. Interestingly, only a daily dose $75 \mathrm{mg}$ of ASC for men and $90 \mathrm{mg}$ of ASC for women is sufficient to prevent scurvy [13]. In 1987, the National Survey of Canada proposed that a serum ASC concentration of $11-23 \mu \mathrm{mol} / \mathrm{L}$ is considered marginally deficient, while a serum ASC concentration of $<11.0 \mu \mathrm{mol} / \mathrm{L}$ is severely deficient; conventions currently used to evaluate ASC levels [5].

\section{Biological Role of Vitamin C}

Deficiency of ASC in the diet leads to scurvy, affecting the skeletal, neurological and hematopoietic systems, thus ASC must be necessary in maintaining normal function of these systems. ASC is a cofactor for hydroxylase enzymes that are responsible for the hydroxylation of proline and lysine residues necessary for the formation of collagen [14]. Collagen lends structural integrity to connective tissues. Using mice with the aldehyde reductase gene knocked-out, an upstream enzyme in the ascorbate biosynthesis pathway relative to Gulo, it was shown that VC administered as a dietary supplement restores osteoblast differentiation and prevents improper proliferation [14]. In other respects, the aldehyde reductase knockout mouse developed scurvy in a manner that is similar to the Gulo knockout. As an antioxidant, ASC also plays an important role in bone formation by 


\section{Genomics \& Gene Therapy International Journal}

modulating the proliferation and death of osteoclasts to maintain levels of bone resorption [10].

In addition to maintaining the structural integrity of bone, the ability to synthesize collagen also reinforces epithelial barriers that are exposed to pathogens, such as the skin and mucosal lining. $\mathrm{VC}$ is found to stimulate leukocyte function during an immune response to infection. An example of VC's role in immunity is its counteraction of oxidative stress brought on by ROS production by neutrophils when exposed to pathogens, alleviating cellular stress and maintaining neutrophil function [15]. VC concentrations in lymphocytes, neutrophils, monocytes and platelets were at saturating levels of $0.4-4 \mathrm{mmol} / \mathrm{L}$ between doses of $200-400 \mathrm{mg} /$ day [13] to protect them from ROS-induced damage. Intravenous administration of vitamin $C$ has previously been shown to decrease inflammation in cancer patients by decreasing the levels of pro-inflammatory cytokines, which are known to promote tumour metastasis and angiogenesis [16].

$\mathrm{VC}$ is a cofactor in the conversion of dopamine to norepinephrine in the catecholamine biosynthesis pathway [3]. It is also involved in neuronal maturation, being detected at high levels in the rat cortex postnatally [17]. Gulo knockout mouse pups deficient in ASC are less mobile and less active than their wild-type counterparts, suggesting striatal dysfunction when insufficiently supplemented with VC [3].

\section{Vitamin C as an Antioxidant}

During oxidative stress, the level of cellular reactive oxygen (ROS) or nitrogen species (RNS) exceeds the antioxidant capacity of the cell. Although ROS are required in modest levels within the body to participate in cellular signalling that regulates normal cell functions such as growth and apoptosis, they are damaging in excess [18]. At high concentrations, these highly reactive species react with macromolecules that comprise cellular components, causing damage if radical propagation is not terminated. An excess of ROS is linked to the induction of cellular autophagy, thus causing neurodegenerative disorders, accelerated aging, cardiovascular disease and cancer.

An important role for $\mathrm{VC}$ is its role as an antioxidant, protecting cells from damage by ROS and RNS generated from metabolism [19]. As an electron donor, ASC acts as a reducing agent that is oxidized in the place of cellular macromolecules, thereby protecting the cell from oxidative damage. The ascorbyl radical generated when ASC loses an electron is stable and will not propagate the reaction, quenching other radicals without causing further cellular damage [20]. The ascorbate species can be regenerated through reduction; however, complete regeneration is incomplete in humans and thus, ascorbate is lost and must be constantly replenished through the diet. Vitamin C supplementation of Gulo knockout mice showed that antioxidant capacity increases linearly with plasma ASC concentration and is responsible for $72 \%$ of the body's total antioxidant capacity [6].

As discussed above, ASC levels are elevated inside leukocytes, to protect them from oxidative damage when metabolic activity increases during an immune response [18]. Several metabolically-active tissues preferentially store ASC such as the lymph tissues, adrenal gland, lung, heart and brain [21]. In particular, the cerebral cortex contained the highest levels of ASC within the brain, such that the cerebral cortex is most sensitive to changes in VC levels when VC supplementation is reduced in the Gulo knockout mouse, resulting in an increase in oxidative stress not seen in other brain regions at the same plasma concentration [22]. In Gulo (-/-) knockout mice, levels of glutathione in the brain increased to compensate for the lack of $\mathrm{VC}$ antioxidant activity to protect the brain. In Gulo knockout mice supplemented with $\mathrm{VC}$, there was a marked increase in oxidative stress compared to wildtype controls [23]. This suggests that there is a benefit of endogenous VC production that cannot be reproduced by VC supplementation. Taken together, these findings suggest that endogenously synthesized VC would avert the development of scurvy in the organism and that consumed VC from the diet or supplementation would increase the levels of $\mathrm{VC}$ in the body beyond physiological levels with supplementation alone.

\section{Direct Administration of VC and Roles of DHASC}

VC, in addition to its role as an essential dietary nutrient, shows potential for the treatment and prevention of disease. A result of ASC being the major physiological form of VC, the activity of its oxidized form, DHASC is much less understood. DHASC is the oxidized form of ASC, and can exert similar physiological effects in mammalian organisms as ASC, and is mostly converted to ASC by DHASC reductase after uptake by the GLUT1 glucose transporter [24,25]. In cases where ASC is required, it may be regenerated intracellularly from DHASC via the glutathione or the thioredoxin systems, as well as through DHASC reductase [26]. ASC, as mentioned above, oxidizes rapidly approximately 12 hours postconsumption in a concentration-dependent manner, while its oxidized form, DHASC maintains stability independent of concentration [27]. Administration of DHASC in place 


\section{Genomics \& Gene Therapy International Journal}

of ASC is sufficient for tissue accumulation of VC levels [27].

As a therapeutic agent, DHASC appears to selectively target cancer cells by competing for glucose uptake via the GLUT1 glucose transporter [24,25]. As a result, when high doses of DHASC are administered, the glutathione stores within the cell are depleted as DHASC is reduced to ASC [25]. Loss of this crucial antioxidant results in an increase of ROS, which inactivates glyceraldehyde-3phosphate dehydrogenase, a key glycolytic enzyme. Combined with a lack of glucose uptake, DHASC is ultimately cytotoxic to cancer cells. In addition, DHASC, but not ASC is able to penetrate the blood brain barrier through GLUT1 [28]. This broadens the therapeutic applicability of DHASC, as VC is implicated in neuronal growth and maturation [17]. The high stability of DHASC makes it a promising therapeutic option, such that it may confer similar benefits as ASC, while remaining in the body for a longer duration. As a result, fewer doses would be required to sustain a therapeutically relevant concentration.

\section{Evolutionary Basis for the Loss of Function in Gulo}

Vertebrate Gulo genes are $64-95 \%$ similar in primary sequence and 11 conserved exons, and strong purifying selection is observed in several lineages, suggesting that vertebrate Gulo genes are highly conserved in these taxa [29]. Select vertebrate taxa such as primates, guinea pigs, bats, teleost fish and passerine birds as reviewed by Drouin, et al. [30]; however, are unable to synthesize VC endogenously due to a loss of the function of the Gulo gene. These animals have different exons deleted from the Gulo coding sequence, which in turn generate premature stop codons. Interestingly, all are left with a nonfunctional protein as a result of different evolutionary processes across species [31]. For example in pigs, deletions in exon VIII and introns 7 and 8 cause a shift in the reading frame of the gene, and leads to a truncated, non-functional protein [2].

Although supplementation is suggested to be the cause of Gulo loss of function, the true reason why loss of function is conserved in so many lineages is unclear and remains controversial [29]. Due to the wide availability of VC in many foods, it is suggested that VC biosynthesis is not inherently beneficial to most animals and there is no selective pressure to maintain a functional pathway [30]. A completely non-functional product, mutations may have accumulated in the Gulo gene without selective pressure, as it served no benefit from these mutations in the context of evolution. Specifically, the mutation occurred at the
Gulo locus prevents upstream precursors from being affected, as these precursors may be used for other pathways [30]. The final product synthesized by Gulo yields VC, an unnecessary product if it can be easily obtained from the diet.

\section{Mouse Models}

To circumvent limitations imposed by a lack of robust models, a Gulo (-/-) knockout mouse was generated [6,32]. Gulo (-/-) knockout mice require dietary supplementation of $\mathrm{VC}$ to maintain redox homeostasis and survive as humans do. Absence of VC supplementation leads to a $30 \%$ reduction in body weight loss by the sixth week, as well as anemia, internal haemorrhaging, hematocrit decrease (-35\%) and haemoglobin decrease $(-37 \%)$. VC supplemented at $330 \mathrm{mg} / \mathrm{L}$ in the drinking water of mice prevented deficiency-induced weight loss and allowed mice to breed normally [6].

Plasma levels of ASC are lower in the Gulo (-/-) knockout mice compared to Gulo $(-/+)$ heterozygote and Gulo $(+/+)$ wildtype mice [6]. Even with dietary supplementation, the level of $\mathrm{VC}$ in the liver was lower in Gulo (-/-) knockout mice than wildtype mice [33]. In other tissues, such as the brain and liver, heterozygotes had slightly, but not significantly lower concentrations of ASC compared to the wildtype [6]. This suggests that the wildtype Gulo allele exhibits dominance over the mutant allele, and that heterozygotes are able to synthesize enough ASC to survive normally. In other respects, hepatic gene expression between VC-supplemented Gulo (-/-) knockout mice and wildtype mice differed, which suggests that the ability to express Gulo may have a broader impact on the metabolism of the organism as a result of the numerous metabolic functions carried out by the liver [33].

\section{Modes of Exogenous Restoration of Gulo Function}

Gene therapy can be used to express exogenous genes ectopically in an organism, or to block the expression of a present gene through gene transfer techniques. This is accomplished with gene transfer vectors such as viruses or nucleic acids. While each vector type has its advantages and disadvantages, it is critical to select the appropriate vector for the gene that is being transferred and the type of model organism.

The use of mRNA as a vector directs the expression of genes, bypassing the transcription step and associated post-transcriptional modification. A limitation of mRNA as 


\section{Genomics \& Gene Therapy International Journal}

a vector, however, is its short half-life, only capable of transient transgene expression [34]. Intramuscular injections of modified erythropoietin mRNA into mice raised hematocrit from $51.5 \%$ to $64.2 \%$ after 28 days [35]. In addition, administration of aerosol mRNA to a knockout mouse model induced expression of surfactant protein SP-B up to $71 \%$ of wildtype levels [35].

DNA as a vector for transgene expression can be in the form of linear cDNA that lacks introns, or circular plasmids. Administered extracellularly, the DNA is taken up into the cell, and used by the host's machinery to transcribe and translate the coding sequences on the construct. DNA can be administered in the form of linear DNA, or circular plasmid DNA isolated from prokaryotic cells both carrying the gene of interest following the appropriate promoters for expression. They are a more stable alternative to mRNA, and a single dose allows the induced gene expression to persist for much longer, despite inefficient uptake into the cell. When singlestranded DNA hairpins flank linear double-stranded DNA, the result is a stable configuration, which enables linear DNA to become a vector for transgene expression [36]. Intramuscularly administered pSO2C1 plasmid in mice with the cry $11 \mathrm{Bb}$ gene from Bacillus thuringiensis caused lifelong expression of the Cry $11 \mathrm{Bb}$ protein and a significant increase in antibody levels, as elevated levels were detected at 119 weeks after injection [37]. The remarkable stability of plasmid DNA vectors and their relative ease to reproduce make them an attractive candidate for gene transfer.

Viruses present a more efficient route of delivery, as they are able to penetrate cell membranes that are virtually impermeable to extracellularly administered nucleic acids. Viruses use the host cell's machinery to express their own genomes after incorporating them into the host genome or maintaining them episomally within host's nucleus. An ideal viral vector is one that has a high infection efficiency of host cells, but modified such that it no longer carries genes that synthesize viral proteins or toxins that are detrimental to the host [38]. Furthermore, these vectors should elicit a minimal host immune response, if at all, to maximize efficiency of delivery without adverse side effects [39]. Taken together, these modes of exogenous Gulo delivery can result in effective expression of functional Gulo both in vivo and in vitro.

\section{Adenovirus-mediated Expression of Gulo}

Adenoviruses are DNA viruses, which inject DNA into host cells and utilize host cellular machinery to express genes [40]. Helper-dependent Adenoviruses (HDAd) do not contain viral genes on their DNA genome and do not integrate their DNA into the host cell genome [41], allowing for highly efficient transgene expression without the interference of viral genes or disruptions to the host genome. They thus rely on a helper virus to propagate, as they lack the necessary viral genes to do so [42]. They are capable of long-term expression; expression of exogenous coagulation factor VIII was detected 2 years postadministration in a canine model of haemophilia [43]. They cause mild reactions, if any, in humans, making them relatively safe and non-pathogenic ). HDAd-mCMV-Gulo adenoviruses were used previously to rescue the expression of Gulo both in vivo and in vitro [7]. Human hepatocellular carcinoma cells (HEPG2) and Gulo (-/-) mice are able to synthesize VC endogenously after transfection with the virus when supplemented with GL.

Expression of Gulo was detected in HEPG2 in a timeand dose-dependent manner upon administration of viruses containing the Gulo construct and supplementation with GL [7]. At concentrations of GL of approximately $4.0 \mathrm{mmol} / \mathrm{L}$ and above, the production of VC reached a plateau of approximately $50 \mathrm{fmol} / \mathrm{cell}$. HEPG2 cells rapidly increased intracellular levels of Gulo expression 24-36 hours after infection, with minimally detectable amounts seen as early as 18 hours postinfection [7]. Levels of Gulo expression increased with the number of viral particles per cell, requiring a minimum of 50 viral particles/cell for Gulo expression to be detectable through Western blot analysis [7].

Serum VC levels increased in Gulo (-/-) knockout mice to levels comparable to that of wildtype mice 4 days after administration of the adenoviruses. Excreted VC in the urine was significantly higher than Gulo (-/-) knockout mice without viruses, but was still significantly lower than that of wildtype mice, suggesting differences in VC retention between the two genotypes [7]. VC production was sustained after administration of the viruses, even after 25 days. Although the level of VC production in the treated Gulo (-/-) knockout mice was lower than the production of wildtype mice, it was still significantly higher than the untreated Gulo (-/-) knockout mice [7]. The results of this study suggest that expression of murine Gulo in human cells is able to rescue the VC biosynthesis pathway.

First generation adenoviruses (FGAd) differ from their HDAd counterparts such that they carry viral genes, and are produced by removing the E1 and E3 regions of the genome and replacing them with an expression cassette, which directs cellular machinery to express the gene of interest [44]. As a result, no helper virus is required for propagation. Like HDAd, these viral vectors rarely integrate viral genes into the host genome, making them relatively safe. However, gene expression is more 


\section{Genomics \& Gene Therapy International Journal}

transient compared to HDAd gene expression [44]. In HEK293Cre cells, incubation with FGAd vectors produced a large increase in Gulo expression, which began to decrease after incubation time exceeded 500 hours, however, levels of expression continued to remain high even after 1500 hours of incubation [45].

\section{Lentiviral-mediated Expression of Gulo}

Lentiviruses are a type of retrovirus that are able to infect both pre- and post-mitotic cells. Lentiviral vectors can facilitate the expression of transgenes in nondividing cells, makes them advantageous over other retroviral vectors that require cell division, as the basis by which viral genes are expressed and propagated [46]. The use of lentiviruses allows for the expression of transgenes that persist and are not silenced through development [47]. Their RNA genomes are reverse-transcribed into DNA and subsequently expressed by the host cell's machinery [47]. Genes can either be silenced through the production of RNAi transcripts, or expressed through the translation of mRNAs [48]. Exogenous genes may be expressed in the organism for up to 24-30 months post-infection [49].

Ongoing work on transgenic Gulo expression focuses on using lentivirus vectors under the murine cytomegalovirus (mCMV) promoter to induce expression of murine Gulo in guinea pigs. Like humans, guinea pigs lack the ability to synthesize VC endogenously, and rely on consuming dietary VC to support health and prevention of scurvy. Administration of the lentivirus extended the lives of guinea pigs maintained on a VC-free diet by 35 days compared to untreated controls that suffered from scurvy. Although ASC was produced in the liver, it was insufficient in elevating plasma levels of ASC. Synthesized ASC was detected in enzyme assays of liver extracts when supplemented with GL. Guinea pigs that received the lentivirus treatment were able to maintain their body weight for longer than the untreated control, with weight declining at a much later time, indicating that the ASC synthesized endogenously was sufficient for supporting some level of enzyme activity and function necessary for survival and prevention of scurvy.

\section{Direct Administration of l-gulono- $\boldsymbol{\gamma}$-lactone Oxidase Enzyme}

At the post-translational level, the Gulo pathway can be restored by direct intraperitoneal injections of Gulo enzyme. Gulo immunoprecipitates allows guinea pigs to synthesize VC when purified with glutaraldehyde to avoid scurvy and lethality [50]. The administration of rat Gulo enzyme rescued the weight loss in guinea pigs associated with VC-starvation after intravenous infusion for four hours, and an increase in body weight was observed up to 4 days after administration [50]. Due to the body's turnover of proteins, long-term expression of Gulo must be administered at regular intervals to replenish the quantity of exogenous Gulo in organisms that cannot express the gene endogenously. Furthermore, the toxicity of immunoprecipitates must be suppressed by reacting the protein with a compound such as glutaraldehyde to stabilize them and prevent their breakdown [50].

Although no recent studies have been performed on Gulo enzyme replacement, enzyme replacement therapy has been employed in more recent years in the treatment of other diseases such as Fabry cardiomyopathy. In Fabry cardiomyopathy, glycosphingolipids accumulate in body tissues due to a lack of the $\alpha$-galactosidase type A enzyme. In a clinical study, the purified enzyme from a human cell line had been intravenously infused at a concentration of $0.2 \mathrm{mg} / \mathrm{kg}$ over a period of one hour every other week for a total of 12 doses [51]. Over this 24-week period, a decrease in pain and associated symptoms of Fabry disease were observed, indicating that enzyme replacement was safe and effective in the treatment of this disease [51]. In the context of Gulo enzyme replacement, the half-life of the synthesized Gulo enzyme must be considered to develop a treatment schedule for enzyme replacement.

\section{Plasmid DNA in Gene Transfer}

Plasmid DNA has been widely used to induce gene expression [52,47,53]. Grown in bacteria, the genes contained on the plasmid confer resistance to certain antibiotics. Recombinant plasmids have been created to insert certain genes and induce their expression in organisms as a vector for gene therapy. They are an attractive alternative to viral vectors, due to their low cost and ease of reproduction [8].

Current venues of research are aimed towards using plasmid DNA therapeutically, for purposes such as conferring resistance to diseases through the expression, effectively silencing or expressing certain genes in an organism and the treatment of cancers [53,54,9]. Mature glycoproteins expressed by pCMV plasmids conferred resistance to lethal Rift Valley fever virus, in addition to inducing lymphoblast proliferation [53]. In another study, plasmids encoding the transgene luciferase free of antibiotic resistance markers (pFARS), demonstrated high levels of transgene expression in mouse muscle, skin and tumour cells (Marie et al., 2010). Naked plasmid DNA injected into the abdominal cavity of rats onto the gastric surface promoted high levels of transgene expression, which can be used in the future for gastric genes as part of 


\section{Genomics \& Gene Therapy International Journal}

a therapeutic regimen for the treatment of gastric ulcers and cancer [9].

Plasmids can be modified to carry functional Gulo, specifically the pSC11 plasmid grown from E. coli DH5 $\alpha$ [7]. The pSC11MC-Gulo plasmid contains the mCMV-GuloPolyA construct, flanked by I-SceI and I-CeuI restriction sites and the pSC11PEPCK-Gulo plasmid containing the PEPCK tissue promoter to induce its expression in tissues. The mCMV viral promoter serves to induce the expression of genes carried by a viral vector. The human CMV immediate-early promoter has the highest level of induced gene expression by adenoviruses in rat hippocampal neurons compared to Rous sarcoma virus and E1A adenoviral promoters, indicating that it is an efficient promoter for inducing the expression of our Gulo plasmid [55]. The mCMV promoter was selected in place of the human cytomegalovirus promoter for this experiment, because it has a higher rate of infection in a murine model, as the human viral promoter demonstrates species specificity [56].

The choice of gene promoter is also important, such that PEPCK was chosen due to its involvement in gluconeogenesis, acting on the glycolysis intermediate phosphoenolpyruvate. PEPCK expression is modulated by cAMP, a ubiquitous second messenger, demonstrated that a cognate coactivator CRCT2 associated with the CREB transcription factor binding site on the PEPCK promoter to enhance PEPCK expression and that exposure to glucagon increased the expression of PEPCK, indicating that gluconeogenesis was taking place. Growth hormone stimulates liver production of PEPCK by inducing STAT5 transcription activator binding to the PEPCK promoter [21]. Taken together, these findings suggest that the PEPCK promoter is capable of inducing gene expression of Gulo in the liver and tissues of the Gulo (-/-) knockout mouse.

\section{Other Approaches to Increase Serum VC Levels}

Other strategies to increase VC levels may include simultaneous administrations of mixed-long chain alcohols which are reported to substantially increase VC levels in patients $[57,58]$ in the absence of $\mathrm{VC}$ supplementation. These mixed-long chain alcohols have also been reported to have other health benefits such as control of blood pressure and treatment of type 2 diabetes. This should be explored further [59].

\section{Future Directions}

The importance of the VC biosynthesis pathway is not only in maintaining physiological homeostasis, but also as a therapeutic venue for the treatment of cancer and other ROS-related illnesses. Available abundantly in normal diet, VC deficiency in most individuals is rare. However, the high rate of $\mathrm{VC}$ excretion prevents above-physiological levels of VC to accumulate in body tissues and thereby blocks its benefits as a therapeutic agent. In the studies discussed above, an elevated level of VC is beneficial for its antioxidant and anti-cancer activity, such that it has the potential to selectively target and kill fast-growing cancer cells.

In the animal models discussed, different modes of ectopic gene expression are efficient in rescuing the ascorbate biosynthetic pathway in both knockout animals and animals endogenously without a functioning pathway. In this context, it shows potential for the elevation of $\mathrm{VC}$ levels in the body by non-dietary means to provide resistance to cancer and ROS-related aging and disease. In the broader scope, the methods of gene therapy discussed above may be applied to other genes that would result in fatality or a decreased quality of life if non-functional, necessitating further research and possible clinical trials. In conclusion, the ectopic expression of genes and the benefits of VC are together and on their own of great benefit to the future of health, as well as disease treatment and prevention.

\section{Acknowledgements}

Research in the Schellhorn Laboratory is supported by the Natural Sciences and Engineering Council (NSERC) of Canada. C. Cao was supported by an NSERC summer

\section{Conflict of Interest}

Cathy Cao, Rachel Kelly, Sulieman Igdoura and Herb Schellhorn declare that they have no conflict of interest.

\section{References}

1. Gabbay KH, Bohren KM, Morello R, Bertin T, Liu J, et al. (2010) Ascorbate Synthesis Pathway. 285: 1951019520.

2. Hasan L, Vögeli P, Stoll P, Kramer SS, Stranzinger G, et al. (2004) Intragenic deletion in the gene encoding Lgulonolactone oxidase causes vitamin $\mathrm{C}$ deficiency in pigs. Mamm Genome 15(4): 323-333.

3. Chen Y, Curran CP, Nebert DW, Patel KV, Williams MT, et al. (2012) Effect of vitamin C deficiency during postnatal development on adult behavior: functional phenotype of Gulo-/- knockout mice. Genes Brain Behav 11(3): 269-277. 


\section{Genomics \& Gene Therapy International Journal}

4. Harrison FE, Dawes SM, Meredith ME, Babaev VR, Li $\mathrm{L}$, et al. (2010) Low vitamin C and increased oxidative stress and cell death in mice that lack the sodiumdependent vitamin C transporter SVCT2. Free Radic Biol Med 49(5): 821-829.

5. Lykkesfeldt J, Poulsen HE (2010) Is vitamin C supplementation beneficial? Lessons learned from randomised controlled trials. Br J Nutr 103(9): 12511259.

6. Maeda N, Hagihara H, Nakata Y, Hiller S, Wilder J, et al. (2000) Aortic wall damage in mice unable to synthesize ascorbic acid. PNAS 97(2): 841-846.

7. Li Y1, Shi CX, Mossman KL, Rosenfeld J, Boo YC, et al. (2008) Restoration of Vitamin C Synthesis in Transgenic Gulo $\chi / \chi$ Mice by Helper-Dependent Adenovirus-Based Expression of Gulonolactone Oxidase. Hum Gene Ther 19(12): 1349-1357.

8. Evans CH (2010) Gene therapy for bone healing. Expert Rev Mol Med 12.

9. Mine T, Ishii H, Nakajima S, Yoshikawa N, Miyamoto $\mathrm{H}$, et al. (2011) Rubbing gastric serosal surface enhances naked plasmid DNA transfer in rats and mice. Biol Pharm Bull 34(9): 1514-1517.

10. Le Nihouannen D, Barralet JE, Fong JE, Komarova S V (2010) Ascorbic acid accelerates osteoclast formation and death. Bone 46(5): 1336-1343.

11. Koshiishi I, Imanari $T$ (1997) Measurement of Ascorbate and Dehydroascorbate Contents in Biological Fluids. Anal Chem 69(2): 216-220.

12. Chambial S, Dwivedi S, Shukla KK, John PJ, Sharma P (2013) Vitamin C in Disease Prevention and Cure : An Overview. Indian J Clin Biochem 28(4): 314-328.

13. Levine M, Wang Y, Padayatty SJ, Morrow J (2001) A new recommended dietary allowance of vitamin $C$ for healthy young women. Proc Natl Acad Sci USA 98(17): 9842-9846.

14. Vissers MC, Bozonet SM, Pearson JF, Braithwaite LJ (2011) Dietary ascorbate intake affects steady state tissue concentrations in vitamin C - deficient mice: tissue deficiency after suboptimal intake and superior bioavailability from a food source (kiwifruit). Am J Clin Nutr 93(2): 292-301.
15. Washko PW, Wang Y, Levine M (1993) Ascorbic acid recycling in human neutrophils. J Biol Chem 268(21): 15531-15535.

16. Mikirova N, Casciari J, Rogers A, Taylor P (2012) Effect of high-dose intravenous vitamin $C$ on inflammation in cancer patients. J Transl Med 10: 189.

17. Terpstra M, Rao R, Tkac I (2010) Region-specific changes in ascorbate concentration during rat brain development quantified by in vivo $1 \mathrm{H}$ NMR spectroscopy. NMR Biomed 23(9): 1038-1043.

18. Bae YS, Oh H, Rhee SG, Yoo Y Do (2011) Regulation of reactive oxygen species generation in cell signaling. Mol Cells 32(6): 491-509.

19. El-Gendy KS, Aly NM, Mahmoud FH, Kenawy A, ElSebae AKH (2010) The role of vitamin C as antioxidant in protection of oxidative stress induced by imidacloprid. Food Chem Toxicol 48(1): 215-221.

20. Padayatty SJ, Katz A, Wang Y, Eck P, Kwon O, et al. (2013) Vitamin $C$ as an Antioxidant: Evaluation of Its Role in Disease Prevention. J Am Coll Nutr 22(1): 1835 .

21. Kim H, Bae S, Yu Y, Kim Y, Kim HR (2012) The Analysis of Vitamin C Concentration in Organs of Gulo - / - Mice Upon Vitamin C Withdrawal. Immune Netw 12(1): 18-26.

22. Harrison FE1, Green RJ, Dawes SM, May JM (2010) Vitamin $C$ distribution and retention in the mouse brain. Brain Res 1348: 181-186.

23. Harrison FE1, Yu SS, Van Den Bossche KL, Li L, May $\mathrm{JM}$, et al. (2008) Elevated oxidative stress and sensorimotor deficits but normal cognition in mice that cannot synthesize ascorbic acid. J Neurochem 106(3): 1198-1208.

24. Wilson JX (2002) The physiological role of dehydroascorbic acid. FEBS Lett 527(1-3): 5-9.

25. Yun J, Mullarky E, Lu C, Bosch KN, Kavalier A, et al. (2016) Vitamin C selectively kills KRAS and BRAF mutant colorectal cancer cells by targeting GAPDH. Science 350(6266): 1391-1396.

26. De Tullio MC, Arrigoni O (2004) Hopes, disillusions and more hopes from vitamin C. Cell Mol Life Sci 61(2): 209-219. 


\section{Genomics \& Gene Therapy International Journal}

27. Frikke-schmidt H, Tveden-nyborg P, Lykkesfeldt J (2016) Redox Biology acid can substitute L -ascorbic acid as dietary vitamin C source in guinea pigs L dehydroascorbic. Redox Biol 7: 8-13.

28. Agus DB, Gambhir SS, Pardridge WM, Spielholz C, Baselga J, et al. (1997) Vitamin C Crosses the Blood Brain Barrier in the Oxidized Form Through the Glucose Transporters. J Clin Invest 100(11): 28422848.

29. Yang H (2013) Conserved or Lost: Molecular Evolution of the Key gene GULO in vertebrate vitamin C biosynthesis. Biochem Genet 51(5-6): 413-425.

30. Drouin G, Godin JR, Pagé B (2011) The Genetics of Vitamin C Loss in Vertebrates. Curr Genomics 12(5): 371-378.

31. Cui J1, Pan YH, Zhang Y, Jones G, Zhang S (2011) Progressive pseudogenization: vitamin $C$ synthesis and its loss in bats. Mol Biol Evol 28(2): 1025-1031.

32. Nishikimi M, Fukuyama R, Minoshima S, Shimizu N, Yagi K (1994) Cloning and chromosomal mapping of the human nonfunctional gene for L-gulono-gammalactone oxidase, the enzyme for L-ascorbic acid biosynthesis missing in man. J Biol Chem 269(18): 13685-13688.

33. Jiao Y, Zhang J, Yan J, Stuart J, Gibson G, et al. (2011) Differential gene expression between wild-type and Gulo-deficient mice supplied with vitamin C. Genet Mol Biol 34(3): 386-395.

34. Smits E, Ponsaerts P, Lenjou M, Nijs G, Van Bockstaele, et al. (2004) RNA-based gene transfer for adult stem cells and T cells. Leukemia 18: 1898-1902.

35. Kormann MS1, Hasenpusch G, Aneja MK, Nica G, Flemmer AW, et al. (2011) Expression of therapeutic proteins after delivery of chemically modified mRNA in mice. Nat Biotechnol 29(2): 154-157.

36. Walters AA, Kinnear E, Shattock RJ, Mcdonald JU, Caproni LJ, et al. (2014) Comparative analysis of enzymatically produced novel linear DNA constructs with plasmids for use as DNA vaccines. Gene Ther 21(7): 645-652.

37. Armengol G, Ruiz LM, Orduz S (2004) The Injection of Plasmid DNA in Mouse Muscle Results in Lifelong Persistence of DNA, Gene Expression, and Humoral Response. Mol Biotechnol 27(2): 109-118.
38. Giacca M, Zacchigna S (2012) Virus-mediated gene delivery for human gene therapy. J Control Release 161(2): 377-388.

39. Croyle MA, Chirmule N, Zhang Y, Wilson JM (2001) "Stealth" adenoviruses blunt cell-mediated and humoral immune responses against the virus and allow for significant gene expression upon readministration in the lung. J Virol 75(10): 47924801.

40. Graham FL, van der Eb AJ (1973) A new technique for the assay of infectivity of human adenovirus 5 DNA. Virology 52(2): 456-467.

41. Vetrini F, Ng P (2010) Gene therapy with helperdependent adenoviral vectors: Current advances and future perspectives. Viruses 2(9): 1886-1917.

42. Rosewell A, Vetrini F, Ng P (2011) Genetic Syndromes \& Gene Therapy Helper-Dependent Adenoviral Vectors. 1-16.

43. McCormack W, Seiler M, Bertin T, Ubhayakar K, Palmer D, et al. (2006) Helper-dependent adenoviral gene therapy mediates long-term correction of the clotting defect in the canine hemophilia A model. J Thromb Haemost 4(6): 1218-1225.

44. Danthinne X, Imperiale M (2000) Production of first generation adenovirus vectors : a review. Gene Ther 7(20): 1707-1714.

45. Ha MN, Graham FL, Souza CKD, Muller WJ, Igdoura SA,et al. (2004) Functional rescue of vitamin C synthesis deficiency in human cells using adenoviralbased expression of murine L -gulono- $g$-lactone oxidase. Genomics 83: 482-492.

46. Naldini L, Blömer U, Gallay P, Ory D, Mulligan R, et al. (1996) In Vivo Gene Delivery and Stable Transduction of Nondividing Cells by a Lentiviral Vector. Science 272(5259): 263-267.

47. Lois C, Hong EJ, Pease S, Brown EJ, Baltimore D (2002) Germline Transmission and Tissue-Specific Expression of Transgenes Delivered by Lentiviral Vectors. Science 295(5556): 868-872.

48. Rubinson DA, Dillon CP, Kwiatkowski AV, Sievers C, Yang L, et al. (2003) A lentivirus-based system to functionally silence genes in primary mammalian cells, stem cells and transgenic mice by RNA interference. Nat Genet 33(3): 401-406. 


\section{Genomics \& Gene Therapy International Journal}

49. Cartier N, Hacein-bey-abina S, Bartholomae CC, Veres G, Schmidt M, et al. (2009) Hematopoietic Stem Cell Gene Therapy with a Lentiviral Vector in X-Linked Adrenoleukodystrophy. Science 326(5954): 818-824.

50. Sato PH, Walton DM (1983) Glutaraldehyde-Reacted Immunoprecipitates Oxidase Are Suitable for Administration of L-Gulonolactone to Guinea Pigs. Arch Biochem Biophys 221(2): 543-547.

51. Schiffmann R, Kopp JB, Iii HAA, Balow JE,Brady RO, et al. (2001) Enzyme Replacement Therapy in Fabry Disease. J Am Med Assoc 285(25): 2743-2749.

52. Kim YD, Li T, Ahn S, Kim D, Lee J, et al. (2012) Orphan Nuclear Receptor Small Heterodimer Partner Negatively Regulates Growth Hormone-mediated Induction of Hepatic Gluconeogenesis through Inhibition of Signal Transducer and Activator of Transcription 5 ( STAT5) transactivation. J Biol Chem 287(44): 37098-37108.

53. Lorenzo G, Martín-Folgar R, Hevia E, Boshra H, Brun A (2010) Protection against lethal Rift Valley fever virus ( RVFV ) infection in transgenic IFNAR $(-/-)$ mice induced by different DNA vaccination regimens. Vaccine 28(17): 2937-2944.
54. Marie C, Vandermeulen G, Quiviger M, Richard M, Préat V, et al (2010) pFARs, Plasmids free of antibiotic resistance markers, display high-level transgene expression in muscle, skin and tumour cells. J Gene Med 12(4): 323-332.

55. Smith RL, Traul DL, Schaack J, Clayton GH, Staley KJ, et al. (2000) Characterization of Promoter Function and Cell-Type-Specific Expression from Viral Vectors in the Nervous System. J Virol 74: 11254-11261.

56. Addison CL, Hitt M, Kunsken D, Graham FL (1997) Comparison of the human versus murine cytomegalovirus immediate early gene promoters for transgene expression by adenoviral vectors. J Gen Virol 78(7): 1653-1661.

57. Raghavan PR (2017a) Vitamins \& Minerals Metadichol @ Induced High Levels of Vitamin C : Case Studies. Vitam Miner 6(4): 169.

58. Raghavan PR (2017b) Metadichol and Vitamin C Increase in vivo, an open-label study. Vitam Miner 6: 163.

59. Vorburger SA, Hunt KK (2002) Adenoviral Gene Therapy. Oncologist 7: 46-59. 